\title{
Prednisone in COPD exacerbation requiring ventilatory support: an open-label randomised evaluation
}

\author{
Fekri Abroug ${ }^{1,2}$, Lamia Ouanes-Besbes ${ }^{1,2}$, Mohamed Fkih-Hassen²,3, \\ Islem Ouanes ${ }^{1,2}$, Samia Ayed ${ }^{2,3}$, Fahmi Dachraoui ${ }^{1,2}$, Laurent Brochard ${ }^{4}$ and \\ Souheil ElAtrous ${ }^{2,3}$
}

Affiliations: 'Intensive Care Unit, Fattouma Bourguiba, University Hospital, Monastir, ${ }^{2}$ Laboratoire de Recherche LR12SP15 "Recherche Cardiopulmonaire en Médecine Intensive et Toxicologie", Ministère de l'Enseignement Supérieur et de la Recherche Scientifique, Tunisia, and ${ }^{3}$ Intensive Care Unit, Tahar Sfar, University Hospital, Mahdia, Tunisia. ${ }^{4}$ ICU Division, Dept of Anesthesiology, Pharmacology and Intensive Care, Geneva University Hospital, Geneva, Switzerland.

Correspondence: F. Abroug, Intensive Care Unit, Fattouma Bourguiba, University Hopsital, 5000 Monastir, Tunisia. E-mail: f.abrougarns.tn

ABSTRACT Recommendation of the use of systemic steroids in chronic obstructive disease (COPD) exacerbation rely on trials that excluded patients requiring ventilatory support.

In an open-label, randomised evaluation of oral prednisone administration, 217 patients with acute COPD exacerbation requiring ventilatory support were randomised (with stratification on the type of ventilation) to usual care $(\mathrm{n}=106)$ or to receive a daily dose of prednisone $\left(1 \mathrm{mg} \cdot \mathrm{kg}^{-1}\right)$ for up to 10 days $(\mathrm{n}=111)$.

There was no difference regarding the primary end-point, intensive care unit mortality, which was 17 (15.3\%) deaths versus 15 (14\%) deaths in the steroid-treated and control groups, respectively (relative risk 1.08, 95\% CI 0.6-2.05). Analysis according to ventilation modalities showed similar mortality rates. Noninvasive ventilation failed in $15.7 \%$ and $12.7 \%$ (relative risk $1.25,95 \%$ CI $0.56-2.8 ; \mathrm{p}=0.59$ ), respectively. Both study groups had similar median mechanical ventilation duration and intensive care unit length of stay, which were 6 (interquartile range 6-12) days versus 6 (3.8-12) days and 9 (6-14) days versus 8 (6-14) days, respectively. Hyperglycaemic episodes requiring initiation or alteration of current insulin doses occurred in $55(49.5 \%)$ patients versus $35(33 \%)$ patients in the prednisone and control groups, respectively (relative risk 1.5, 95\% CI 1.08-2.08; $\mathrm{p}=0.015$ ).

Prednisone did not improve intensive care unit mortality or patient-centred outcomes in the selected subgroup of COPD patients with severe exacerbation but significantly increased the risk of hyperglycaemia.

@ERSpublications

In COPD exacerbation needing ventilatory support, prednisone has no impact on ICU mortality or related patient outcome http://ow.ly/qxGq0

For editorial comments see page 668 .

Received: Jan 062013 | Accepted after revision: May 072013 | First published online: June 212013

Clinical trial: This study is registered at www.clinicaltrials.gov with identifier number NCT01353235.

Conflict of interest: None declared.

Copyright (OERS 2014 


\section{Introduction}

Chronic obstructive pulmonary disease (COPD) is a condition of chronic airflow limitation that is not completely reversible and is often progressive [1]. By 2010, it had become the third leading cause of mortality worldwide [2]. The natural course of COPD is characterised by the occurrence of exacerbations (usually two to three per year) requiring either an emergency visit to hospital or hospitalisation [3-5]. Acute exacerbations of COPD (AECOPD) are not only responsible for the largest part of the economic burden associated with COPD, but they also accelerate the decline in lung function and worsen the prognosis of the disease with elevated in-hospital and 1-year mortalities (11\% and 40\%, respectively), and have a 6-month relapse rate of 50\% [6-10]. COPD exacerbations are usually associated with increases in local and systemic inflammatory response, and are treated with systemic steroids in accordance with high-grade recommendations [3,11-13]. Indeed, although data are insufficient to define the optimal dose, route or duration of systemic corticosteroids, current guidelines strongly recommend administration of systemic steroids (prednisone equivalent doses of 30-40 mg $\cdot$ day $^{-1}$ ) to hospitalised patients with AECOPD [1, 14, 15]. Recommendations rely on meta-analyses with cumulated effects showing both a significant reduction in the rate of treatment failure, and an increase in the rate of improvement in lung function and dyspnoea [16-18]. However, systemic corticosteroids were not associated with a reduction in the mortality rate, and induced a significant increase in adverse effects (in particular a five-fold increase in hyperglycaemic episodes) $[17,18]$. Moreover, primary studies included in these meta-analyses usually excluded COPD patients with an exacerbation severe enough to require ventilatory support in the intensive care unit (ICU). It is therefore unclear whether recommendations derived from studies that systematically excluded patients with severe AECOPD requiring ventilatory support should be extrapolated to this type of patient, especially knowing that corticosteroid administration to critically ill patients might be associated with severe adverse events, such as infections, muscle paresis, hyperglycaemia and other metabolic disorders. These side-effects cause increased morbidity and mortality. In the COPD population in particular, hyperglycaemic episodes were clearly associated with poor outcomes with an increased rate of noninvasive ventilation (NIV) failure $[19,20]$.

In this context of paucity of evidence, AliA et al. [21] have recently published the only study dealing specifically with patients suffering from severe COPD exacerbation requiring ventilatory support. This multicentre Spanish randomised study included 83 patients and evaluated the effects of a 10-day course of intravenous methylprednisolone. Compared with placebo, corticosteroids reduced the duration of mechanical ventilation by 1 day, and reduced the risk of failure of NIV by $93 \%$. Conversely, steroid treatment had no impact on ICU mortality, and induced a two-fold increase in hyperglycaemic episodes.

The current study reports a prospective, open-label, randomised evaluation of oral prednisone administration in acute COPD exacerbation requiring ventilatory support.

\section{Methods}

This randomised controlled trial with two parallel groups was conducted between 2008 and 2011 in two Tunisian ICUs belonging to two tertiary teaching hospitals: CHU Fattouma Bourguiba, Monastir and CHU Tahar Sfar, Mahdia, both of which affiliated with the University of Monastir. The trial was approved by the ethics committee of both participating centres, and written informed consent was obtained from either the patients or their surrogates.

\section{Inclusion criteria}

All patients aged $\geqslant 40$ years, with a history of at least 10 pack-years of cigarette smoking, and with known or strongly suspected COPD, who were admitted to participating ICUs for an AECOPD with hypercapnic acute respiratory failure requiring ventilatory support, were considered for inclusion in the study. COPD, COPD exacerbation and respiratory failure were defined according to the Global Initiative for Chronic Obstructive Lung Disease (GOLD) criteria [1]. COPD is defined as a preventable and treatable disease whose pulmonary component is characterised by airflow limitation that is not fully reversible. Airflow limitation was deemed to be present if the post-bronchodilator ratio of forced expiratory volume in $1 \mathrm{~s}$ $(\mathrm{FEV} 1)$ /forced vital capacity (FVC) ratio was $<0.7$. Patients with suspected COPD and without previous documentation of the FEV1/FVC ratio had pulmonary function tests checked on discharge from the ICU. COPD exacerbation corresponds to a change in patient's baseline dyspnoea, cough and/or sputum requiring a change in regular medication. Acute respiratory failure is defined by severe hypoxaemia (arterial oxygen tension $\left(\mathrm{PaO}_{2}\right)<60 \mathrm{mmHg}$ and/or arterial oxygen saturation $<90 \%$ on room air) associated with hypercapnia (with arterial carbon dioxide tension $\left(\mathrm{PaCO}_{2}\right) \geqslant 45 \mathrm{mmHg}$ associated with $\mathrm{pH} \leqslant 7.35$ ) and clinical signs of excessive respiratory muscle activity (contraction of accessory respiratory muscles and respiration rate $\geqslant 25$ breaths $\cdot \mathrm{min}^{-1}$ ). 


\section{Noninclusion criteria}

Patients were not included if they had evidence of pneumonia, were treated for COPD exacerbation with systemic steroids within 30 days prior to screening or had an absolute contraindication to steroids (active gastroduodenal ulcer, severe uncontrolled sepsis, hepatitis or other active viral disease and/or neuromuscular disease).

\section{Methods}

In addition to ventilatory support, all included patients received nebulisation of $\beta_{2}$-agonists (terbutaline, $5 \mathrm{mg}$ every $6 \mathrm{~h}$ ) and ipratropium bromide $(0.5 \mathrm{mg}$ every $8 \mathrm{~h})$. Antibiotics were prescribed at the discretion of physician in charge. Patients were randomised (by means of sealed envelopes that were opened sequentially) to receive prednisone as an add-on therapy to usual treatment. Randomisation was performed at each centre by a random number table, and was stratified according to the type of mechanical ventilation (either conventional or noninvasive). Patients assigned to the corticosteroid treatment arm received oral prednisone $1 \mathrm{mg} \cdot \mathrm{kg}^{-1}$ daily either until discharge or for a maximum of 10 days. Prednisone was administered within $24 \mathrm{~h}$ of admission to the ICU as a single dose in the morning. In patients on conventional mechanical ventilation, the tablets were administered via a feeding tube.

In patients who were initially treated with NIV, treatment was deemed to be failing (and patients considered to need tracheal intubation) if any of the following major criteria were present: hypercapnia with respiratory acidosis $\left(\mathrm{pH} \leqslant 7.20\right.$ and below its value at inclusion); hypercapnic coma (Glasgow Coma Scale 8 and $\mathrm{PaCO}_{2}$ $\geqslant 60 \mathrm{mmHg}$ ); $\mathrm{PaO}_{2}<45 \mathrm{mmHg}$ despite a maximum tolerated inspiratory oxygen fraction; and/or cardiac arrest. In intubated patients, weaning from mechanical ventilation was started after daily screening to evaluate their recovery from respiratory failure and their eligibility to the weaning process. Patients were considered ready to be weaned from mechanical ventilation when they met the following weaning criteria: $\mathrm{PaO}_{2}$ /inspiratory oxygen fraction $\geqslant 150 \mathrm{mmHg}$; an effective cough; no vasopressors and no sedation and a Glasgow Coma Score $\geqslant 12$. Weaning began with a spontaneous breathing trial (SBT) on a T-tube for $2 \mathrm{~h}$. Patients who tolerated the SBT were subsequently extubated. SBT was deemed to be failing when any of the following signs of intolerance were present: agitation, altered neurological status, cyanosis, contraction of accessory respiratory muscles, thoraco-abdominal dyssynchrony, tachypnoea, tachycardia or arrhythmia.

NIV was considered successful and stopped if the initial NIV criteria were no longer present while breathing without ventilatory assistance for at least $4 \mathrm{~h}$.

\section{End-points}

The primary end-point was ICU mortality. The secondary end-points were the lengths of ventilatory support (the sum of conventional and NIV in those ventilated with both) and length of ICU stay. The rate of NIV failure (inferred from the intubation rate in patients managed initially with NIV) was also compared between study groups. Corticosteroidal complications were evaluated on the occurrence of secondary infections, hyperglycaemic episodes necessitating initiation of insulin therapy (corresponding to a blood glucose level $\geqslant 180 \mathrm{mg} \cdot \mathrm{dL}^{-1}$ in patients without pre-existing diabetes) or increase in initial insulin therapy, ICU-acquired muscular weakness, or significant gastrointestinal bleeding inducing a fall in the haemoglobin level $\geqslant 2 \mathrm{~g} \cdot \mathrm{dL}^{-1}$.

\section{Statistical analyses}

Data are presented as either median (interquartile range (IQR)) or proportion, as appropriate. The study groups were compared on an intention-to-treat basis, and a p-value $\leqslant 0.05$ was considered significant. Continuous variables were compared with the Mann-Whitney test. Categorical variables were compared using the Chi-squared test. Based on the mortality rate we previously reported in this type of patient $(22 \%$ in the control group), we calculated that it would be necessary to include 150 patients in each arm of the study in order to detect a $12 \%$ reduction in the absolute risk of mortality with a power of $80 \%$ and a twotailed $\alpha$ risk of 0.05 [22]. SPSS (version 17; SPSS, Chicago, IL, USA) was used for statistical analyses.

\section{Results}

During the study period, 518 patients were admitted in both participating ICUs for severe COPD exacerbation requiring ventilatory support. Of these, 217 (42\%) fulfilled the inclusion criteria and were included in the study and randomised to either an active treatment group $(n=111)$ or a control group $(n=106)$. The study was ended before completion of the planned sample size because of the slow inclusion rate. The flow chart of the study is reported in figure 1 . The main cause of noninclusion was treatment with systemic corticosteroids within the preceding 30 days, or the presence of pneumonia as a cause of exacerbation. There was no difference in baseline characteristics of included and nonincluded patients, in particular with regard to age, Simplified Acute Physiology Score (SAPS) II and pH. 


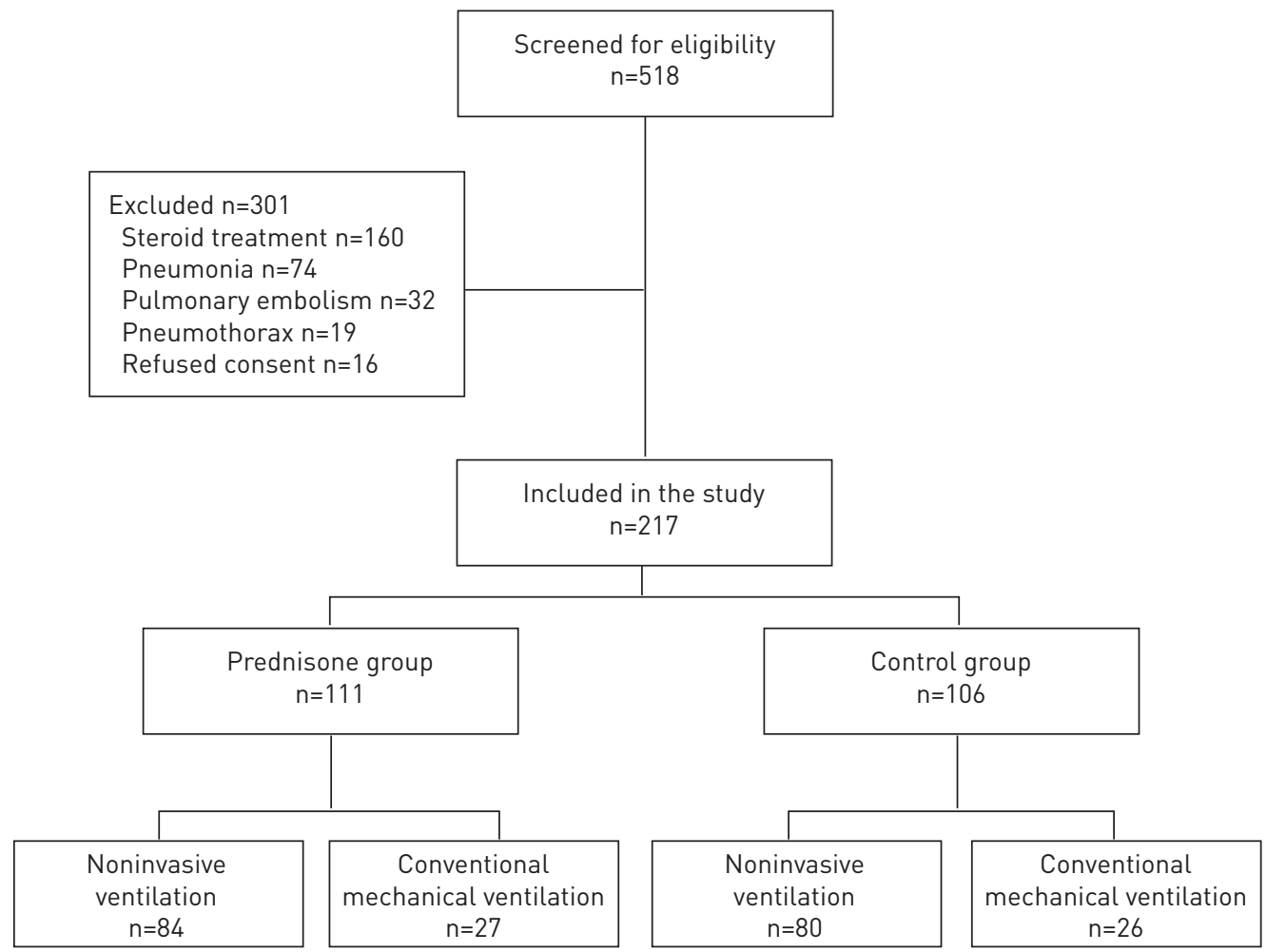

FIGURE 1 Study flow chart.

Baseline characteristics of both study groups are reported in table 1. Treatment and control groups were similar with respect to demographics, COPD severity (70\% and $73 \%$ of GOLD stage IV, respectively), frequencies of comorbidities, and the severity of acute exacerbation as reflected by SAPS II (29 (24-35) versus 27 (20-35)) and $\mathrm{pH}(7.28$ (7.25-7.32) versus 7.29 (7.25-7.32), respectively).

Similar rates of NIV $(76 \%)$ were applied as initial ventilation mode in both groups. Prednisone was administered for a median of 8 (IQR 5-10) days in the active treatment group. Its administration started at a median of 9 (IQR 6-13) h, following the admission to ICU. All patients received nebulised terbutaline and ipratropium. The use of systemic antibiotics was not statistically different: antibiotics (mainly cephalosporins in association with either one quinolone or macrolide) were administered in $82 \%$ and $79 \%$ of patients in the steroid-treated and control groups, respectively, and for a median duration of 7 days.

\section{Outcomes}

There was no statistically significant difference between patients treated with prednisone and the control group with regard to the main end-point, namely mortality in the ICU (table 2): 17 (15.3\%) deaths versus $15(14.2 \%)$ deaths, respectively (relative risk $1.08,95 \%$ CI $0.6-2.05 ; \mathrm{p}=0.81$ ). Similar mortality rates were also found in noninvasively ventilated patients: $10.5 \%$ versus $11.3 \%$, (relative risk $0.93,95 \%$ CI $0.37-2.35$; $\mathrm{p}=0.88$ ) in the steroid-treated and control groups, respectively. In the subgroup of patients who had conventional ventilation, ICU mortality occurred in $25.7 \%$ and $20.0 \%$ of the prednisone and control groups, respectively (relative risk 1.28 , 95\% CI $0.54-3$; p $=0.57$ ).

NIV failed in $12(15.7 \%)$ patients and nine (12.7\%) patients in steroid-treated and control groups, respectively (relative risk $1.25,95 \%$ CI $0.56-2.8 ; \mathrm{p}=0.59$ ). None of the study patients was intubated due to cardiac arrest. Both study arms had similar mechanical ventilation duration and ICU length of stay; 6 (4-12) days versus 6 (3.8-12) days and 9 (6-14) days versus 8 (6-14) days, respectively.

No episode of ICU-acquired muscle weakness or gastrointestinal bleeding occurred in the study population. Ventilator-associated pneumonia episodes occurred in five and four patients in the steroid-treated and control groups, respectively. Hyperglycaemic episodes requiring initiation or alteration of current insulin doses occurred in $55(49.5 \%)$ patients versus 35 patients (33\%) in the steroid-treated and control groups, respectively (relative risk 1.5, 95\% CI 1.08-2.08; $\mathrm{p}=0.015$ ). 
TABLE 1 Baseline characteristics of the study patients

\begin{tabular}{|c|c|c|}
\hline & Prednisone & Control \\
\hline Subjects & 111 & 106 \\
\hline Age years & $70(63-75)$ & $68(63-75)$ \\
\hline Males & $99(89)$ & 92 (87) \\
\hline Baseline FEV $1 \mathrm{~mL} \cdot \mathrm{s}^{-1}$ & $820(590-1120)$ & $750(565-950)$ \\
\hline COPD duration years & $10(5-12)$ & $8(5-12.5)$ \\
\hline Home oxygen therapy & $80(72)$ & $71(67)$ \\
\hline \multicolumn{3}{|l|}{ Comorbidities } \\
\hline History of diabetes & $17(15)$ & $13(12)$ \\
\hline History of hypertension & $9(8)$ & 7 (7) \\
\hline History of heart failure & $14(13)$ & $13(12)$ \\
\hline \multicolumn{3}{|l|}{ GOLD stage } \\
\hline III & $33(30)$ & $29(27)$ \\
\hline IV & $78(70)$ & 77 (73) \\
\hline \multicolumn{3}{|l|}{ Cause of AECOPD } \\
\hline Respiratory tract infection & $50(45)$ & $46(43)$ \\
\hline Heart dysfunction & $34(31)$ & $32(30)$ \\
\hline Unidentified & $27(24)$ & $28(27)$ \\
\hline SAPS II score & $29(24-35)$ & $27(20-35)$ \\
\hline Respiratory rate breaths $\cdot \min ^{-1}$ & $30(25-34)$ & $29(24-34)$ \\
\hline \multicolumn{3}{|l|}{ Arterial blood gases } \\
\hline $\mathrm{pH}$ & $7.28(7.25-7.32)$ & $7.29(7.25-7.32)$ \\
\hline $\mathrm{PaCO}_{2} \mathrm{mmHg}$ & $78(62-85)$ & $80(62-87)$ \\
\hline $\mathrm{SaO}_{2} \%$ & $90(84-94)$ & $89(83-94)$ \\
\hline $\mathrm{CRP} \mathrm{mg} \cdot \mathrm{L}^{-1}$ & $24(12-57)$ & $37(12-66)$ \\
\hline \multicolumn{3}{|l|}{ Initial ventilatory support } \\
\hline NIV & $84(76)$ & $80(76)$ \\
\hline Conventional & $27(24)$ & $26(24)$ \\
\hline
\end{tabular}

Data are presented as $\mathrm{n}$, median (interquartile range) or $\mathrm{n}(\%)$. FEV1: forced expiratory volume in $1 \mathrm{~s}$; COPD: chronic obstructive pulmonary disease; GOLD: Global Initiative for Chronic Obstructive Lung Disease; AECOPD: acute exacerbation of COPD; SAPS II: Simplified Acute Physiology Score II; $\mathrm{PaCO}_{2}$ : arterial carbon dioxide tension; $\mathrm{SaO}_{2}$ : arterial oxygen saturation; CRP: C-reactive protein; NIV: noninvasive ventilation.

\section{Discussion}

In this prospective open-label controlled study, the evaluated regimen of oral prednisone $\left(1 \mathrm{mg} \cdot \mathrm{kg}^{-1}\right.$ daily for up to 10 days) in patients admitted to the ICU for COPD exacerbation requiring ventilatory support had no effect on patient-centred outcomes, such as ICU mortality, duration of mechanical ventilation, length of ICU stay or the rate of NIV failure. Prednisone administration was associated with a significant increase in the rate of hyperglycaemic episodes requiring initiation or alteration of current insulin treatment. Given the established evidence on the negative impact of hyperglycaemic episodes on the outcome of critically ill patients in general, and in particular those admitted for AECOPD, our study does not argue for the systematic administration of corticosteroids in severe COPD exacerbation requiring ICU admission and ventilatory support.

The present study was stopped before its completion because of a slow inclusion rate. Although it achieved $70 \%$ power to detect the $12 \%$ assigned lowering in the absolute risk of ICU mortality, the current study was underpowered and should not be considered a definitive negative study. Indeed, the lower boundary of the confidence interval for the effect on mortality cannot preclude a lowering by $40 \%$ in the relative risk of ICU mortality. According to the mortality rate observed herein, a prospective randomised trial would take no less than 2000 patients to detect the minimum clinically important reduction in mortality and draw definitive conclusions regarding the effects of systemic corticosteroids on mortality. Similar numbers are also needed for the evaluation of another patient-centred outcome, such as NIV failure. The open-label design of a randomised clinical trial may elicit bias in reporting potential end-points. However, the fact that our study focused on the so-called "hard" end-points increases confidence in observed results. Notwithstanding these limitations, the results of our study actually challenge the findings of ALIA et al. [21] and the explicit recommendations of systematic administration of systemic steroids in COPD exacerbation $[4,11,21]$. Regarding patients admitted to ICUs, most of these recommendations could be 
TABLE 2 Efficacy and safety end-points

Prednisone

ICU mortality in patients ventilated conventionally

\section{Secondary end-points}

NIV failure

Mechanical ventilation duration days ICU length of stay days

\section{Safety end-point}

Hyperglycaemic episodes requiring initiation or alteration of insulin therapy

\section{Control}

$17 / 111(15.3)$
$8 / 76(10.5)$

9/35 (25.7)

$15 / 106(14.2)$
$8 / 71(11.3)$

$7 / 35(20.0)$

$9 / 71(12.7)$

6 (3.8-12)

$6(4-12)$

$9(6-14)$

$55 / 111(49.5)$
$8(6-14)$

$35 / 106$ (33.0)
Relative risk $(95 \% \mathrm{CI})$

p-value

Data are presented as $\mathrm{n} / \mathrm{N}(\%)$ or median (interquartile range), unless otherwise stated. ICU: intensive care unit, NIV: noninvasive ventilation.

regarded as not fully supported as they rely on studies that explicitly excluded patients requiring ICU admission and ventilatory support $[14,17,23,24]$.

The only available study that dealt specifically with patients requiring ventilatory support, has recently been published by AliA et al. [21], who conducted a multicentre double-blind placebo-controlled trial evaluating a 10-day course of intravenous methylprednisolone in patients with severe COPD exacerbation. Patients were randomised to receive either intravenous methylprednisolone $\left(2 \mathrm{mg} \cdot \mathrm{kg}^{-1}\right.$ for 3 days, $1 \mathrm{mg} \cdot \mathrm{kg}^{-1}$ for the following 3 days and then $0.5 \mathrm{mg} \cdot \mathrm{kg}^{-1}$ for the remaining 4 days) or placebo. The main outcome measure was the mechanical ventilation duration, and a sample size of 198 patients was deemed necessary in order to reduce mechanical ventilation duration by 2 days. Owing to a low inclusion rate precluding the study completion, only 83 patients were eventually included in the trial (43 in the active treatment group and 40 in the placebo group). Still, the authors reported a small but statistically significant reduction by one day in the mechanical ventilation duration (from 4 days in the control group to 3 days in the steroid group). Steroid treatment only marginally impacted on both the ICU mortality and the length of stay. The most striking effect of steroid treatment in this study was observed among the subgroup of 37 patients who received NIV with a reduction in the NIV failure rate: 0 out of 18 in the steroid treatment group compared with seven (37\%) out of 19 (relative risk 0.07 ) in the control group. Although this effect seems clinically relevant, the small number of patients in whom this was observed should make one cautious when interpreting these results, which deserve further confirmation. NIV was indeed used as a first ventilatory mode in only $44 \%$ compared with $76 \%$ of patients in our study.

The apparent contradiction between our results and those of AliA et al. [21] should not be readily explained by the difference in the chosen scheme of steroid administration. Only differences pertaining to the initial corticoid dose and administration route could actually be considered relevant and might account for the apparently discrepant results. In the study by AliA et al. [21], patients received (during the first 3 days via the intravenous route) a prednisone equivalent dose twice that received by our patients. However, issues like steroid daily dose, the route of administration and the course duration are poorly standardised in the treatment of COPD exacerbation. Different doses (low initial dose versus higher doses) [18], route of administration (by mouth or intravenously) $[25,26]$ and course duration (3 days, 7 days or even beyond) [27] have been used in previous studies, and little, if any, evidence suggests that these parameters could have a drastic impact on steroids efficacy. For the particular issue of initial steroid dose, a meta-analytic comparison of high initial dose (i.e. $\geqslant 80 \mathrm{mg}$ prednisone equivalent dose) and low initial dose of systemic steroids showed no superiority of the high-dose regime over the low-dose regime [18]. With regard to these considerations, we do not see a major design difference between our study and that of AliA et al. [21], and potential causes of differences, including the effect on NIV failure, must be sought elsewhere. As acknowledged by the authors, the fact that NIV failed in none of the 18 noninvasively ventilated patients from the steroid group accounted for a greater impact on mechanical ventilation duration in the NIV subgroup (minus 2 days of mechanical ventilation in this subgroup), and could explain the statistically significant reduction of the mechanical ventilation duration by 1 day in the overall population. In fact, NIV failure rates reported by AliA et al. [21], either those observed in the control group (37\%) or those recorded 
in the intervention group $(0 \%)$, are very dissimilar to the average $(16 \%)$ reported to date in the literature [28]. As a matter of fact, the impact of steroid treatment on the NIV failure rate might suggest that, despite randomisation, two populations with different inflammatory and biological characteristics were actually included in each study arm in the randomised clinical trial by ALIA et al. [21]. AECOPD is indeed heterogeneous with respect to inflammation, which is most often predominantly neutrophilic but can be eosinophilic in a significant number of instances $[29,30]$. Patients with eosinophilic inflammation behave like asthmatic patients, with a strong expression in bronchospastic exacerbations and a high potential for reversibility under corticosteroid treatment. Clinically, these patients are very difficult to distinguish from others at the time of exacerbation. Auscultation of wheezing only reflects bronchial obstruction and does not indicate its potential for reversibility. The measurement of FEV1 and a reversibility test under inhalation of bronchodilators is virtually impossible to perform in such dyspnoeic patients. Some studies have shown that sputum eosinophilia can predict a positive response to corticosteroids treatment in stable COPD or can be used to titrate maintenance steroid treatment [31-33]. The eosinophilic pattern of inflammation in COPD exacerbation is also reflected by an increase in peripheral blood eosinophil count [30], and BAFADHEL et al. [34] have recently validated the use of peripheral blood eosinophil count to guide prescription of systemic corticosteroids during AECOPD. The different results of the study by ALIA et al. [21] and our study suggest that different subpopulations were enrolled and one may hypothesise that a strategy of corticosteroid prescribing based on peripheral blood or sputum eosinophil count might be an interesting approach, considering the risk-benefit balance in this population. Testing such an approach could help to better determine a specific subpopulation of patients with COPD exacerbation who could benefit from systemic corticosteroids.

Even if the level of statistical significance was not achieved, the effects of steroid administration on the primary outcome, ICU mortality, seem quite different in patients ventilated noninvasively (relative risk 0.93 ) compared with the intubated population where the relative risk of ICU mortality is increased by $28 \%$ (relative risk 1.28). We cannot readily account for these statistical trends, but we can speculate that in intubated patients, systemic steroids may alter the general prognosis either by favouring ventilator-associated pneumonia or by inducing a higher rate of hyperglycaemic episodes. The reduced sample of the subgroup of intubated patients in our study precludes such post hoc analysis, which has a limited scientific value.

We see no impediment or bias that could prevent the extrapolation of the results of this two-centre study to other patients/settings. The clinical characteristics, management and outcomes of the patients included herein are similar to those generally reported in severe exacerbation of COPD requiring ICU admission and ventilatory support. This is particularly seen through the severity of the index episode (inferred from median SAPS and $\mathrm{pH}$ at inclusion), the rates of NIV use and failure, and rates of mortality, which are in the levels usually reported in studies dealing with severe COPD exacerbation [21, 35-38].

In summary, administration of steroids in patients with severe episodes of COPD exacerbation and requiring ventilatory support failed to demonstrate any benefit, did not alter the rate of NIV failure and resulted in more frequent episodes of hyperglycaemia. These results do not support recommending this approach until a more precise identification of potential responders is possible.

\section{References}

1 Global Initiative for Chronic Obstructive Lung Disease (GOLD). Global Strategy for the Diagnosis, Management and Prevention of COPD. 2013. www.goldcopd.org Date last accessed: July 2, 2013.

2 Lozano R, Naghavi M, Foreman K, et al. Global and regional mortality from 235 causes of death for 20 age groups in 1990 and 2010: a systematic analysis for the Global Burden of Disease Study 2010. Lancet 2012; 380: 2095-2128. Wedzicha JA, Seemungal TA. COPD exacerbations: defining their cause and prevention. Lancet 2007; 370: 786-796.

Celli BR, MacNee W. ATS/ERS Task Force Report: Standards for the diagnosis and treatment of patients with COPD: a summary of the ATS/ERS position paper. Eur Respir J 2004; 23: 932-946.

5 Buist AS, McBurnie MA, Vollmer WM, et al. International variation in the prevalence of COPD (the BOLD Study): a population-based prevalence study. Lancet 2007; 370: 741-750.

6 Connors AF Jr, Dawson NV, Thomas C, et al. Outcomes following acute exacerbation of severe chronic obstructive lung disease. The SUPPORT investigators (Study to Understand Prognoses and Preferences for Outcomes and Risks of Treatments). Am J Respir Crit Care Med 1996; 154: 959-967.

7 Pinto-Plata VM, Cote C, Cabral H, et al. The 6-min walk distance: change over time and value as a predictor of survival in severe COPD. Eur Respir J 2004; 23: 28-33.

8 Strassels SA, Smith DH, Sullivan SD, et al. The costs of treating COPD in the United States. Chest 2001; 119: 344-352.

9 Wedzicha JA, Wilkinson T. Impact of chronic obstructive pulmonary disease exacerbations on patients and payers. Proc Am Thorac Soc 2006; 3: 218-221.

10 Donaldson GC, Seemungal TA, Bhowmik A, et al. Relationship between exacerbation frequency and lung function decline in chronic obstructive pulmonary disease. Thorax 2002; 57: 847-852.

11 O’Donnell DE, Aaron S, Bourbeau J, et al. Canadian Thoracic Society recommendations for management of chronic obstructive pulmonary disease - 2007 update. Can Respir J 2007; 14: Suppl. B, 5B-32B. 
Perera WR, Hurst JR, Wilkinson TM, et al. Inflammatory changes, recovery and recurrence at COPD exacerbation. Eur Respir J 2007; 29: 527-534.

13 Fujimoto K, Yasuo M, Urushibata K, et al. Airway inflammation during stable and acutely exacerbated chronic obstructive pulmonary disease. Eur Respir J 2005; 25: 640-646.

14 Sayiner A, Aytemur ZA, Cirit M, et al. Systemic glucocorticoids in severe exacerbations of COPD. Chest 2001; 119: 726-730.

15 Pizzichini MM, Pizzichini E, Efthimiadis A, et al. Asthma and natural colds. Inflammatory indices in induced sputum: a feasibility study. Am J Respir Crit Care Med 1998; 158: 1178-1184.

16 Wood-Baker R, Walters J, Walters EH. Systemic corticosteroids in chronic obstructive pulmonary disease: an overview of Cochrane systematic reviews. Respir Med 2007; 101: 371-377.

17 Walters JA, Gibson PG, Wood-Baker R, et al. Systemic corticosteroids for acute exacerbations of chronic obstructive pulmonary disease. Cochrane Database Syst Rev 2009; 1: CD001288.

18 Cheng T, Gong Y, Guo Y, et al. Systemic corticosteroid for COPD exacerbations, whether the higher dose is better? A meta-analysis of randomized controlled trials. Clin Respir J 2012 [In press DOI: 10.1111/cri.12008].

19 Chakrabarti B, Angus RM, Agarwal S, et al. Hyperglycaemia as a predictor of outcome during non-invasive ventilation in decompensated COPD. Thorax 2009; 64: 857-862.

20 Baker EH, Bell D. Blood glucose: of emerging importance in COPD exacerbations. Thorax 2009; 64: 830-832.

21 Alia I, de la Cal MA, Esteban A, et al. Efficacy of corticosteroid therapy in patients with an acute exacerbation of chronic obstructive pulmonary disease receiving ventilatory support. Arch Intern Med 2011; 171: $1939-1946$.

22 Nouira S, Marghli S, Belghith M, et al. Once daily oral ofloxacin in chronic obstructive pulmonary disease exacerbation requiring mechanical ventilation: a randomised placebo-controlled trial. Lancet 2001; 358: $2020-2025$.

23 Maltais F, Ostinelli J, Bourbeau J, et al. Comparison of nebulized budesonide and oral prednisolone with placebo in the treatment of acute exacerbations of chronic obstructive pulmonary disease: a randomized controlled trial. $A m \mathrm{~J}$ Respir Crit Care Med 2002; 165: 698-703.

24 Bullard MJ, Liaw SJ, Tsai YH, et al. Early corticosteroid use in acute exacerbations of chronic airflow obstruction. Am J Emerg Med 1996; 14: 139-143.

25 Lindenauer PK, Pekow PS, Lahti MC, et al. Association of corticosteroid dose and route of administration with risk of treatment failure in acute exacerbation of chronic obstructive pulmonary disease. JAMA 2010; 303: $2359-2367$.

26 de Jong YP, Uil SM, Grotjohan HP, et al. Oral or IV prednisolone in the treatment of COPD exacerbations: a randomized, controlled, double-blind study. Chest 2007; 132: 1741-1747.

27 Walters JA, Wang W, Morley C, et al. Different durations of corticosteroid therapy for exacerbations of chronic obstructive pulmonary disease. Cochrane Database Syst Rev 2011; 10: CD006897.

28 Ram FS, Picot J, Lightowler J, et al. Non-invasive positive pressure ventilation for treatment of respiratory failure due to exacerbations of chronic obstructive pulmonary disease. Cochrane Database Syst Rev 2004; 3: CD004104.

29 Saetta M, Di Stefano A, Maestrelli P, et al. Airway eosinophilia in chronic bronchitis during exacerbations. Am J Respir Crit Care Med 1994; 150: 1646-1652.

30 Bafadhel M, McKenna S, Terry S, et al. Acute exacerbations of chronic obstructive pulmonary disease: identification of biologic clusters and their biomarkers. Am J Respir Crit Care Med 2011; 184: 662-671.

31 Pizzichini E, Pizzichini MM, Gibson P, et al. Sputum eosinophilia predicts benefit from prednisone in smokers with chronic obstructive bronchitis. Am J Respir Crit Care Med 1998; 158: 1511-1517.

32 Brightling CE, Monteiro W, Ward R, et al. Sputum eosinophilia and short-term response to prednisolone in chronic obstructive pulmonary disease: a randomised controlled trial. Lancet 2000; 356: 1480-1485.

33 Siva R, Green RH, Brightling CE, et al. Eosinophilic airway inflammation and exacerbations of COPD: a randomised controlled trial. Eur Respir J 2007; 29: 906-913.

34 Bafadhel M, McKenna S, Terry S, et al. Blood eosinophils to direct corticosteroid treatment of exacerbations of chronic obstructive pulmonary disease: a randomized placebo-controlled trial. Am J Respir Crit Care Med 2012; 186: 48-55.

35 Maggiore SM, Richard JC, Abroug F, et al. A multicenter, randomized trial of noninvasive ventilation with heliumoxygen mixture in exacerbations of chronic obstructive lung disease. Crit Care Med 2010; 38: 145-151.

36 Esteban A, Ferguson ND, Meade MO, et al. Evolution of mechanical ventilation in response to clinical research. Am J Respir Crit Care Med 2008; 177: 170-177.

37 Abroug F, Ouanes-Besbes L, Nciri N, et al. Association of left-heart dysfunction with severe exacerbation of chronic obstructive pulmonary disease: diagnostic performance of cardiac biomarkers. Am J Respir Crit Care Med 2006; 174: 990-996.

38 Scala R, Nava S, Conti G, et al. Noninvasive versus conventional ventilation to treat hypercapnic encephalopathy in chronic obstructive pulmonary disease. Intensive Care Med 2007; 33: 2101-2108. 